\title{
Bioinformatics analysis of aberrantly expressed exosomal IncRNAs in oral squamous cell carcinoma (CAL-27 vs. oral epithelial) cells
}

\author{
JIAJIA JIN ${ }^{1,2^{*}}$, ZIXIAO HUANG $^{1 *}$, XIAOYAN LU ${ }^{1}$, SHENGRONG WU ${ }^{1}$, \\ MEI'E JIA ${ }^{1}, \mathrm{XIN} \mathrm{LI}^{1}, \mathrm{ZHIYONG} \mathrm{LI}^{3}$ and XIANGYI HE${ }^{1,4}$
}

\begin{abstract}
${ }^{1}$ Department of Prosthodontics, School of Dentistry, Lanzhou University, Lanzhou, Gansu 730000; ${ }^{2}$ Department of Stomatology, Xi'an Daxing Hospital, Xi'an, Shaanxi 710000; ${ }^{3}$ State Key Laboratory of Veterinary Etiological Biology, Lanzhou Veterinary Research Institute, Chinese Academy of Agricultural Sciences, Lanzhou, Gansu 730046; ${ }^{4}$ Group of Molecular Biology, Key Laboratory of Functional Genomic and Molecular Diagnosis of Gansu Province, Lanzhou, Gansu 730030, P.R. China
\end{abstract}

Received August 29, 2019; Accepted May 27, 2020

DOI: 10.3892/ol.2020.11764

\begin{abstract}
Oral squamous cell carcinoma (OSCC) is the most prevalent form of malignant tumour in the oral cavity and its early detection is critical for improving the prognosis of affected patients. The present study aimed to isolate and confirm exosomes derived from the supernatant of the OSCC cell line CAL-27 and human oral epithelial cells (HOECs), analyze long non-coding RNA (lncRNA) expression profiles and determine the diagnostic value based on bioinformatics analyses. The results indicated that the particles isolated from the supernatant of CAL-27 and HOECs were either round or oval, had a size range of 30-150 $\mathrm{nm}$ and were enriched with ALG-2 interacting protein X (ALIX) and tumour susceptibility 101 proteins (TSG101). These characteristics confirmed that these particles were exosomes. Three lncRNAs (NR-026892.1, NR-126435.1 and NR-036586.1) were selected as potential diagnostic biomarkers for OSCC. The expression levels of the selected lncRNAs were significantly different in CAL-27-exo vs. HOEC-exo, as well as in whole cells (CAL-27 vs. HOECs $)(\mathrm{P}<0.001)$. The expression levels of the three
\end{abstract}

Correspondence to: Professor Xiangyi He, Department of Prosthodontics, School of Dentistry, Lanzhou University, 199 Donggang Xi Lu, Chengguan, Lanzhou, Gansu 730000, P.R. China E-mail: hexy@lzu.edu.cn

Dr Zhiyong Li, State Key Laboratory of Veterinary Etiological Biology, Lanzhou Veterinary Research Institute, Chinese Academy of Agricultural Sciences, 1 Xujiaping, Chengguan, Lanzhou, Gansu 730046, P.R. China

E-mail: lizhiyong02@caas.cn

*Contributed equally

Key words: oral squamous cell carcinoma, exosome, long non-coding RNAs, bioinformatics
lncRNAs confirmed by quantitative PCR were consistent with the sequencing data. In conclusion, various lncRNAs were aberrantly expressed between cancerous and non-cancerous exosomes, suggesting that they may serve as biomarkers for cancer.

\section{Introduction}

Oral squamous cell carcinoma (OSCC) accounts for $>90 \%$ of oral and maxillofacial cancers and for $3 \%$ of all malignant types of tumour (1). Tissue biopsies and their histological analysis are conventionally the gold standard in diagnosing OSCC and determining its type and stage based on which to make clinical management decisions; however, limitations such as invasive and difficult to perform sampling methods, as well as and time-consuming analysis, are primary challenges $(2,3)$. With the rapid development of molecular biological technology, previous studies have focused on early detection and sensitive noninvasive biomarkers for OSCC diagnosis $(1,4)$.

Exosomes are microvesicles with a lipid bilayer membrane; their size ranges from 30 to $150 \mathrm{~nm}$ and their density is $1.13-1.19 \mathrm{~g} / \mathrm{ml}$ of sucrose. Exosomes can be identified using the specific membrane marker ALG-2 interacting protein X (ALIX) and tumor susceptibility 101 proteins (TSG101). Studies have indicated that exosomes may be abundantly released by different cell types into physiological fluids, such as the peripheral blood, urine and breast milk (5). Depending on the type, biogenesis and physiological condition of cells, exosomes contain a wide range of proteins, lipids and nucleic acids, reflecting the characteristics of parental cells (6). In exosomes, nucleic acids [including microRNAs (miRNAs/miRs) and long non-coding RNAs (lncRNAs)] are protected from rapid degradation by a lipid bilayer membrane to maintain their stability; they may be transported into target cells to remain stable and may be regulated over long distances (7). Therefore, exosomes are regarded as an entirely new type of cell-to-cell communication; their cellular messengers trigger a variety of physiological and pathological 
responses and achieve cross-talk to adjacent or distant cells (8).

lncRNAs are a class of ncRNAs with $>200$ nucleotides. Although lncRNAs have been initially considered 'transcriptional noise', they have been regarded as emerging regulators of biological functions and described to have an essential role in gene expression at multiple levels, such as chromatin remodelling, $\mathrm{X}$ chromosome silencing and transcriptional activation; they also participate in cell growth, differentiation, metabolism and oncogenesis (9). Studies have also indicated that aberrant lncRNA expression is associated with numerous cancer types, e.g., colorectal cancer, breast cancer and liver cancer (10). Therefore, differential expression of exosomal RNA (exo-RNA), including miRNAs and lncRNAs, may exist in various cancer types $(11,12)$.

A considerable amount of studies has focused on the aberrant expression of exo-miRNAs in OSCC (13-15), but only a few studies have explored exo-lncRNAs $(16,17)$. Thus, the present study aimed to investigate exo-lncRNA expression profiles and their detection potential for OSCC through a bioinformatics analysis.

\section{Materials and methods}

Cell isolation and culture. Human oral epithelial cells (HOECs) were isolated and used in accordance with the ethical standards established in the Declaration of Helsinki. The present study was approved by the ethics committee of the School/Hospital of Stomatology at Lanzhou University (Lanzhou, China). HOECs were isolated and cultured as previously described (18-20). Normal oral mucosa was sampled and pooled between March and June 2018 from 10 patients (4 females and 6 males) who had surgery for cleft lip reconstruction (age range, 0.3-1 years; mean age, 0.78 years) and did not have any tumours. Informed consent forms were signed by the patients' parents prior to their participation in the study. The specimens were washed using sterile PBS containing penicillin and streptomycin. After the connective tissue was removed, the specimens were cut into small $2 \mathrm{~mm} \times 1 \mathrm{~mm}$ pieces and incubated in Dispase II (Merck Millipore) at $4^{\circ} \mathrm{C}$ for $18 \mathrm{~h}$. The epithelial layer was mechanically separated, digested with $0.25 \%$ trypsin $/ 0.02 \%$ EDTA (Thermo Fisher Scientific, Inc.) at $37^{\circ} \mathrm{C}$ for $7 \mathrm{~min}$ and vigorously mixed. The mixture was collected in a tube and centrifuged at $100 \mathrm{xg}$ for $7 \mathrm{~min}$ at $4^{\circ} \mathrm{C}$. The supernatant was carefully removed, and the cells were resuspended in an EpiGROTM Human Epidermal Keratinocyte Expansion Medium (Merck Millipore). The cell line CAL-27 was obtained from the American Type Culture Collection and cultured in Dulbecco's modified Eagle's medium (DMEM) supplemented with $10 \%(\mathrm{v} / \mathrm{v})$ fetal bovine serum (FBS; both Gibco; Thermo Fisher Scientific, Inc.). Once the cells grew in a stable manner, they were washed with PBS and the CAL-27 medium was replaced with DMEM supplemented with $10 \%$ (v/v) exosome-depleted FBS (System Biosciences). All of the supernatants were collected and maintained for 2 days at $4^{\circ} \mathrm{C}$ for further analysis.

Exosome isolation. As described in a previous study by our group, the general approach of isolating exosomes from the cell culture supernatant was based on differential ultracentrifugation. Once the volume of the collected supernatant reached $100 \mathrm{ml}$, all the following operations were performed at $4^{\circ} \mathrm{C}$ : The supernatant was first centrifuged at $300 \mathrm{x} \mathrm{g}$ for $10 \mathrm{~min}$, then at 2,000 $\mathrm{xg}$ for $10 \mathrm{~min}$ and finally at $10,000 \mathrm{x} \mathrm{g}$ for $30 \mathrm{~min}$ to discard the pellets (cells, dead cells and cell debris). The supernatant was then ultracentrifuged at $100,000 \times \mathrm{g}$ for $70 \mathrm{~min}$ at $4^{\circ} \mathrm{C}$ to produce a sediment and concentrate the exosomes. The pellet was resuspended in PBS and collected in a centrifuge tube. The sample was centrifuged at $100,000 \mathrm{xg}$ for $70 \mathrm{~min}$ at $4^{\circ} \mathrm{C}$ and the supernatant was carefully removed by using a pipette. The pellet after centrifugation was washed with PBS, whilst the pellet containing the exosomes was resuspended in $100 \mu \mathrm{l} \mathrm{PBS}$ and maintained at $-80^{\circ} \mathrm{C}$ for further analysis.

After the particles were isolated from CAL-27 and HOECs, the morphological characteristics and phenotype of the particles were identified using the procedures described below.

Western blot analysis. The particles were thawed and treated with radioimmunoprecipitation assay buffer (Beyotime Institute of Biotechnology) and protease inhibitor cocktail (Beyotime Institute of Biotechnology) in accordance with the manufacturer's protocols. Subsequently, 2X SDS-PAGE loading buffer (Sigma-Aldrich; Merck KGaA) was added, followed by heating to $95^{\circ} \mathrm{C}$ for $5 \mathrm{~min}$. The proteins were separated using $10 \%$ SDS-PAGE and transferred onto nitrocellulose (NC) membranes (Beyotime Institute of Biotechnology). The NC membranes were blocked with $5 \%$ skimmed milk powder at room temperature for $1.5 \mathrm{~h}$, washed thrice with TBS-Tween (0.1\% Tween-20), by using an orbital shaker and incubated with primary antibodies at $4^{\circ} \mathrm{C}$ overnight. The following primary antibodies were used: Rabbit monoclonal anti-ALIX (1:1,000 dilution; cat. no. F-2609; System Biosciences) and goat monoclonal anti-TSG 101 (1:1,000 dilution; cat. no. K-1711; System Biosciences). Subsequently, the NC membranes were incubated with horseradish peroxidase-conjugated secondary goat anti-mouse antibody (1:5,000 dilution; System Biosciences) and rabbit anti-mouse antibody (1:5,000 dilution; System Biosciences) at room temperature for $1 \mathrm{~h}$. The membranes were visualized using Immobilon Western Chemiluminescent HRP Substrate (cat. no. WBKLS0100; EMD Millipore) on a Bio-Rad gel imaging system (Gel Doc ${ }^{\mathrm{TM}}$ $\mathrm{XR}^{+}$; Bio-Rad Laboratories, Inc.).

Transmission electron microscopy. Isolated particles were resuspended in PBS and $20 \mu \mathrm{l}$ of the mixture was loaded onto Formvar-coated 200-mesh copper electron microscopy grids at room temperature for $10 \mathrm{~min}$. Excess liquid was drained by touching the grid edge against a piece of filter paper. Counterstaining was performed by floating the grid on a drop of $3 \%$ phosphotungstic acid solution ( $\mathrm{pH}$ 6.8) at room temperature for $5 \mathrm{~min}$. Excess liquid was drained and the grid was dried for several minutes at room temperature. Microphotographs were obtained using an HT7700 transmission electron microscope (Hitachi).

Particle size analysis. The particles isolated from $100 \mathrm{ml}$ collected supernatant were thoroughly resuspended in $1 \mathrm{ml}$ PBS. The size distribution of the particles was determined 
A

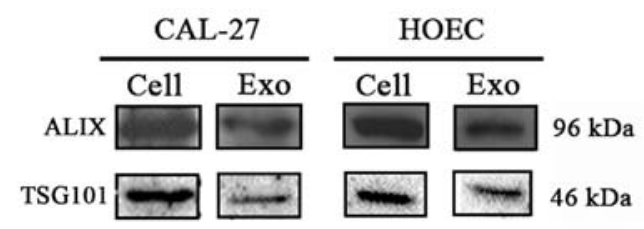

B
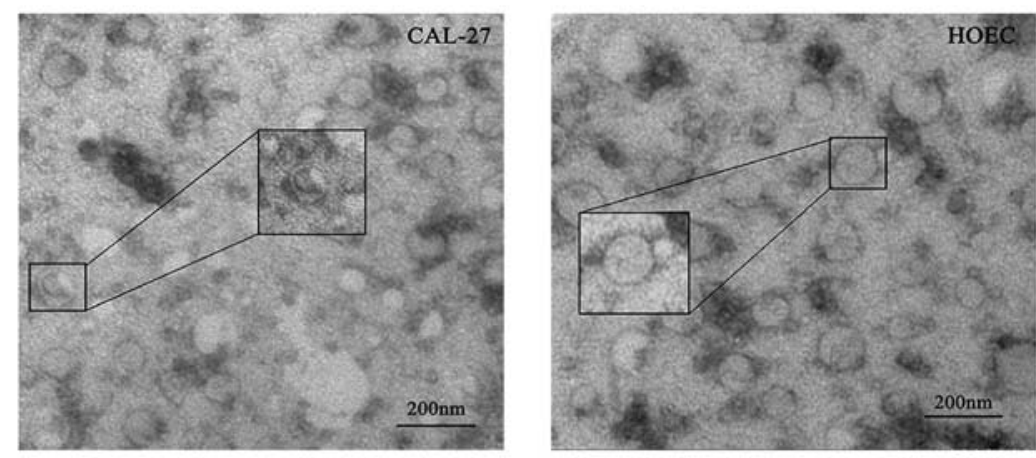

C
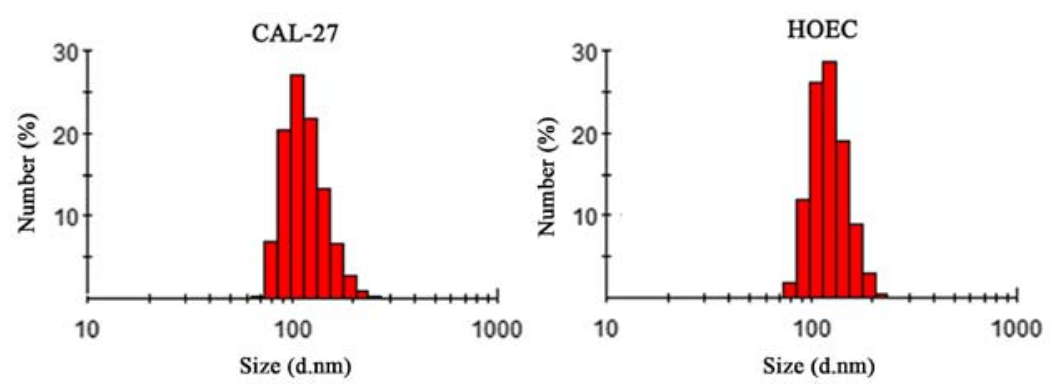

Figure 1. Exosome identification. (A) Western blot analysis of exosome markers (samples with different exposures); (B) transmission electron microscopy images of exosomes (magnification, x30,000; scale bar, $200 \mathrm{~nm}$ ); (C) Zetasizer Nano ZS analysis of the mean size of exosomes ( 100 nm). ALIX, ALG-2 interacting protein X; TSG101, tumor susceptibility 101; HOEC, human oral epithelial cell; exo, exosome; d, diameter.

using a Zetasizer Nano ZS (Malvern) in accordance with the manufacturer's protocols.

High-throughput lncRNA sequencing. Following the manufacturer's protocol, the total RNA of exosomes was extracted using the Total Exosome RNA and Protein Isolation kit (Invitrogen; Thermo Fisher Scientific, Inc.). The total RNA of exosomes was used for IncRNA library preparation and sequencing, which was performed at RiboBio Co., Ltd., where the total RNAs from CAL-27-exo and HOEC-exo were reverse-transcribed and their ends were repaired and amplified through PCR. The PCR products were sequenced using the Illumina HiSeq 2500 (Illumina, Inc.). Raw data were initially filtered in terms of low-quality reads, ribosomal RNA contamination and reads containing $>10 \%$ uncertain nucleotides. Subsequently, clean reads were generated and mapped with the reference genome by using TopHat 2 for the subsequent analysis (21).

Bioinformatics analysis. After the sequencing data were obtained, Auddics was used as a classic procedure to identify differential RNA expression. The target genes of lncRNAs were predicted using GENCODE (https://www.gencodegenes. org/), the European Molecular Biology Laboratory-European Bioinformatics Institute (https://www.ebi.ac.uk/), lncRNAdb (http://www.lncrnadb.org/) and the gene database from the National Center for Biotechnology Information (https://www. ncbi.nlm.nih.gov/) according to their cis or trans mecha- nism to systematically uncover the functions of lncRNAs. Their comprehensive functions were analysed using Gene Oncology (GO) and Kyoto Encyclopedia of Genes and Genomes (KEGG) (http://www.genome.jp/). Furthermore, the $\mid \log _{2}$ (fold-change) $\mid>2$, significance level $(\mathrm{P}<0.05)$ and cancer-associated pathways of IncRNA-targeted genes were set to select the candidate lncRNAs and evaluate their diagnostic potential.

Relative expression levels of the selected lncRNAs. The relative expression of the selected lncRNAs was assessed using reverse transcription-quantitative (RT-q)PCR to further validate the data from the high-throughput lncRNA sequencing. The total RNAs were extracted from the cells and exosomes using an RNeasy Mini kit (Qiagen $\mathrm{GmbH}$ ) and the Total Exosome RNA and Protein Isolation kit (Invitrogen; Thermo Fisher Scientific, Inc.), respectively. The RNAs were treated with RNase-free DNase I (Takara Bio, Inc.) to remove any DNA contamination and eluted in $25 \mu 1$ R Nase-free ultrapure water. The relative lncRNA expression was determined using PrimeScript ${ }^{\mathrm{TM}}$ RT Master Mix (Perfect Real Time; Takara Bio, Inc.) and SYBR-Green ${ }^{\mathrm{TM}}$ Premix Ex Taq $^{\mathrm{TM}}$ II (Tli RNaseH Plus; Takara Bio, Inc.) on a 7500 sequence detector system (Applied Biosystems; Thermo Fisher Scientific, Inc.). PCR was performed in a mixture (20 $\mu \mathrm{l}$ ) containing $2 \mu \mathrm{l}$ of complementary DNA template, $10 \mu 12$ X SYBR-Green PCR Mix, $0.4 \mu 1$ Rox II and $0.8 \mu 1$ each of sense and antisense primers. RT-qPCR was performed in 
A

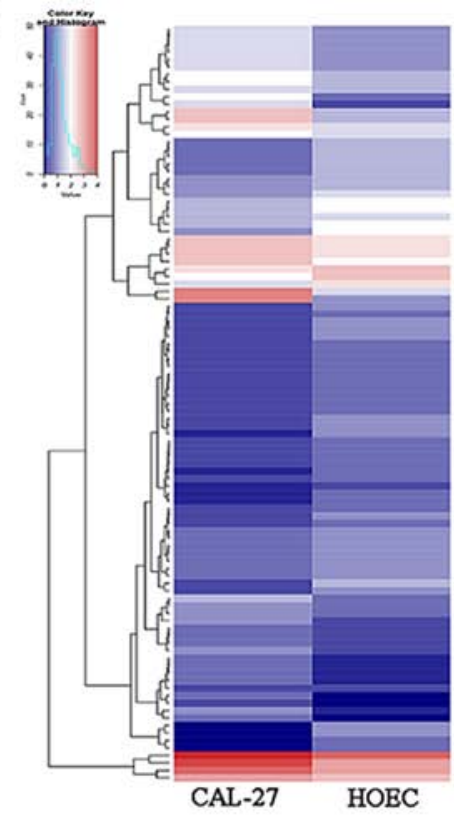

C

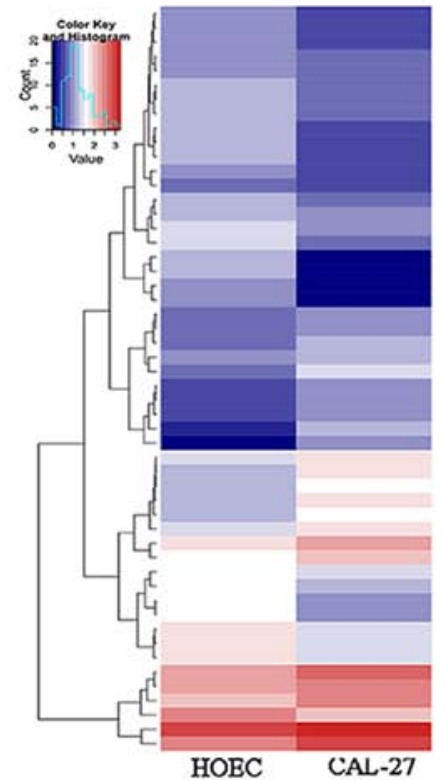

B

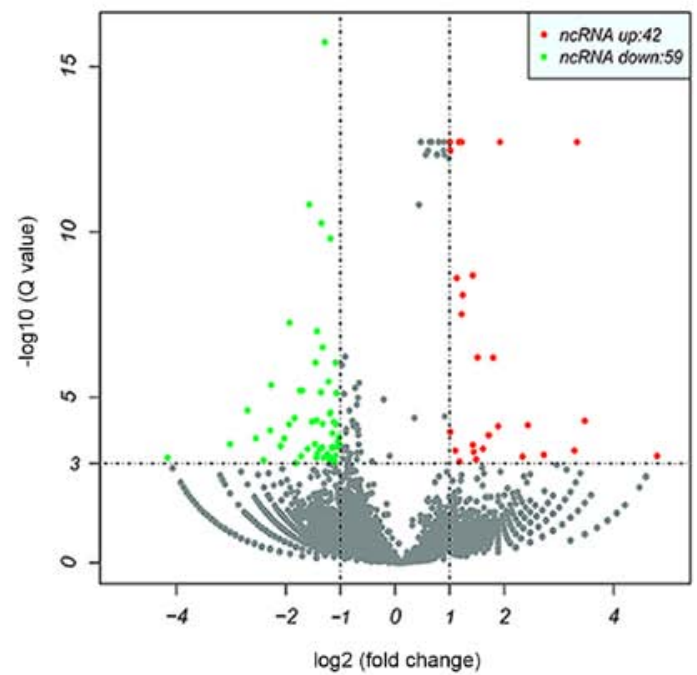

D

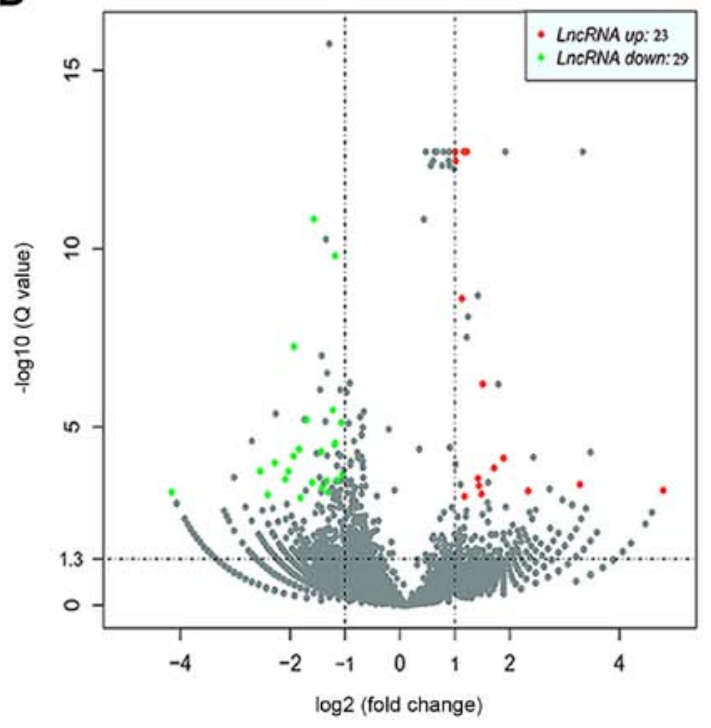

Figure 2. Hierarchical clustering and volcano plots of differentially expressed ncRNAs and lncRNAs between HOEC-exo and CAL-27-exo. (A) Hierarchical clustering analysis of differentially expressed ncRNAs; each column represents a sample and each row indicates an lncRNA. The colour indicates the value of $\log _{10}$ (RPKM+1). (B) Volcano plot of differentially expressed ncRNAs; the abscissa refers to the fold-change of ncRNA and lncRNA expression in different samples and the ordinate corresponds to the significance of the differential expression of the ncRNAs and lncRNAs. (C) Hierarchical clustering analysis of differentially expressed lncRNAs; each column represents a sample and each row indicates an lncRNA. The colour indicates the value of $\log _{10}(\mathrm{RPKM}+1)$ (D) Volcano plot of differentially expressed lncRNAs; the abscissa refers to the fold-change of ncRNA and lncRNA expression in different samples and the ordinate corresponds to the significance of the differential expression of the ncRNAs and lncRNAs. Red dots denote significant upregulation of ncRNAs and lncRNAs and green dots represent significant downregulation of ncRNAs and lncRNAs. HOEC, human oral epithelial cell; lncRNA, long non-coding RNA; exo, exosome; RPKM, Reads Per Kilobase per Million mapped reads.

triplicate for each sample. GAPDH was used as the control and the specificity of the PCR products was estimated from the melting curve. The following primer sequences used for qPCR were synthesized by Tsingke Biological Technology Co., Ltd.: N $\quad \mathrm{N}_{-} 026892.1 \quad$ forward, 5'-GGTCTACCAGTTGCACAG ATT-3' and reverse, 5'-CAGAGAAAGAAGGTGGG AGTTAG-3'; NR_036586.1 forward, 5'-CCAACATGGGC TCTCAATACA-3' and reverse, 5'-CACCATACCTGGCACA TACAA-3'; NR_126435.1 forward, 5'-GTCTGACATCCA
GAGCCAATAC-3' and reverse, 5'-AGGCCTAACCATGTT TCCTTAC-3'; and GAPDH forward, 5'-GGTGAAGGTCGG AGTCAACGG-3' and reverse, 5'-GAGGTCAATGAAGGG GTCATTG-3'. The relative expression of each lncRNA was calculated using the $2^{-\Delta \Delta \mathrm{Cq}}$ method (22).

Statistical analysis. Data were presented as the mean \pm SD $(n=3)$. GraphPad Prism version 6.0 (GraphPad Software, Inc.) was used for all of the calculations. An unpaired Student's t-test was applied to examine the differences in lncRNA expression 
A

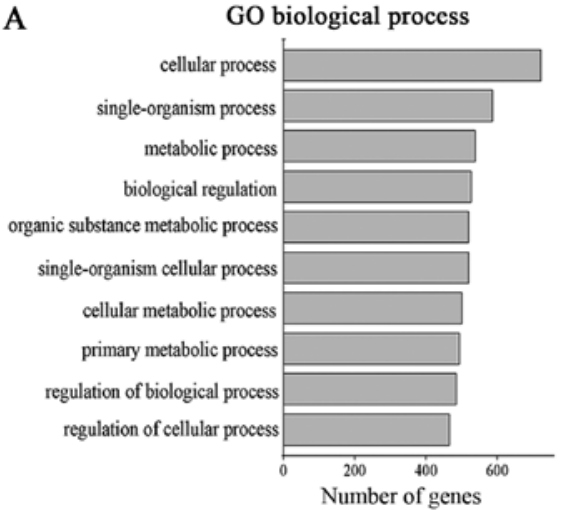

C

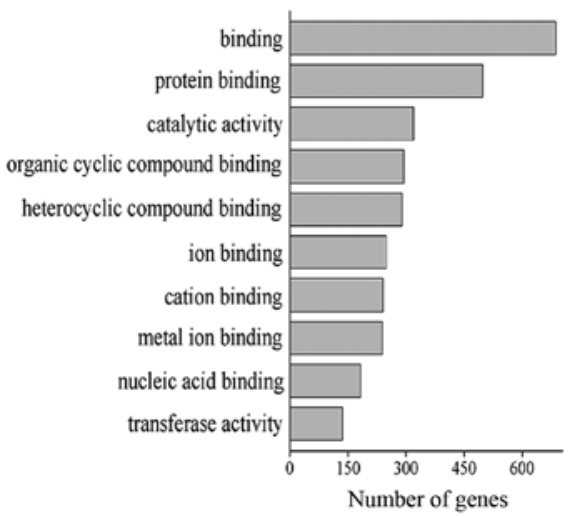

B

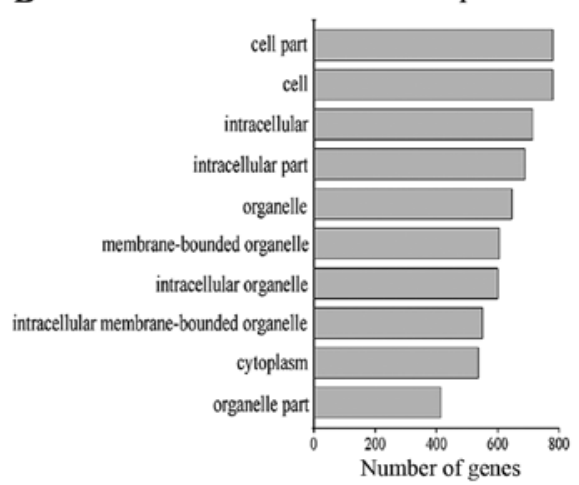

D

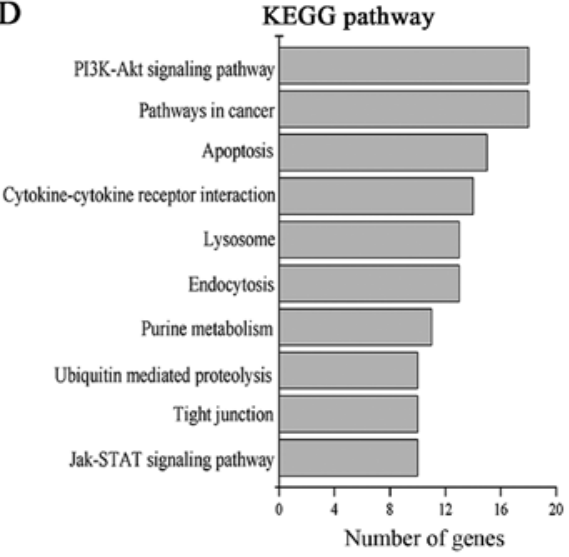

Figure 3. GO terms and KEGG pathway analysis with respect to the target genes of lncRNAs. (A-C) GO annotation in the categories (A) biological process, (B) cellular component and (C) molecular function. (D) KEGG pathways. GO, gene ontology; KEGG, Kyoto Encyclopedia of Genes and Genomes; lncRNA, long non-coding RNA.

obtained via RT-qPCR. $\mathrm{P}<0.05$ was considered to indicate a statistically significant difference.

\section{Results}

Exosomes from CAL-27 and HOEC culture supernatant. The particles isolated from the supernatant of CAL-27 and HOEC were verified by detecting the expression of ALIX and TSG101, which are known markers of exosomes (23) (Fig. 1A). The exosomes had a round or oval shape and a membrane structure (Fig. 1B). The size of most particles ranged from 30 to $150 \mathrm{~nm}$ and the cumulative percentages of the particle diameter interval for CAL-27 in the ranges of 0-30, 30-150 and $>150 \mathrm{~nm}$ were $0,89.6$ and $10.4 \%$, respectively, and those for HOEC were $0,87.8$ and $12.4 \%$, respectively (Fig. 1C). These observations confirmed that the particles isolated from the supernatant were exosomes.

ncRNA and lncRNA expression in exosomes. The sequencing data identified 28,437 ncRNAs in CAL-27 and 29,254 ncRNAs in HOECs. By using the parameters difference multiple ( $\log _{2}$ (fold-change) $\mid>1$ ), false discovery rate $(\mathrm{FDR})<0.001$ and $\mathrm{P}<0.05$, a total of 101 differentially expressed ncRNAs between CAL-27-exo and HOEC-exo were identified. Amongst them, 42 ncRNAs were upregulated, whereas 59 ncRNAs were downregulated (Fig. 2A and B). Of the 101 ncRNAs, 52 differentially expressed lncRNAs were identified with 23 upregulated lncRNAs and 29 downregulated lncRNAs (Fig. 2C and D).
Functional enrichment analysis of the target genes of differentially expressed lncRNAs. GO annotation in the categories biological process, cellular component and molecular function was performed. A total of 1,549 GO terms in the biological process category $(\mathrm{P}<0.05), 227 \mathrm{GO}$ terms in the cellular component category $(\mathrm{P}<0.05)$ and $320 \mathrm{GO}$ terms in the molecular function category $(\mathrm{P}<0.05)$ were identified. The top $10 \mathrm{GO}$ terms of each category are presented in Fig. 3A-C, respectively; 'cell' and 'cell part' were the most prominent terms in the cellular component category, while terms in the molecular function and biological process categories were mainly associated with cellular processes, binding functions and catalytic activity. KEGG pathway analysis identified 50 pathways $(\mathrm{P}<0.05)$. The top $10 \mathrm{KEGG}$ pathways are presented in Fig. 3D. The target genes of lncRNAs were mainly associated with the regulation of the 'PI3K/Akt signaling pathway', 'Jak/STAT signaling pathway', 'pathways in cancer' and 'apoptosis'. These data confirmed that exosomal lncRNAs may be involved in cell metabolism and oncogenesis in general.

Selection and functional prediction of candidate lncRNAs. After 52 differentially expressed lncRNAs were screened, the expression levels of $12 \operatorname{lncRNAs}$ were identified to match $\log _{2}$ (fold-change) $\mid>2, F D R<0.001$ and $\mathrm{P}<0.05$. These $12 \operatorname{lncRNAs}$ were further filtered if their target genes were involved in tumour-associated pathways and 3 IncRNAs (i.e., NR-026892.1, NR-126435.1 and NR-036586.1) were ultimately selected as the 

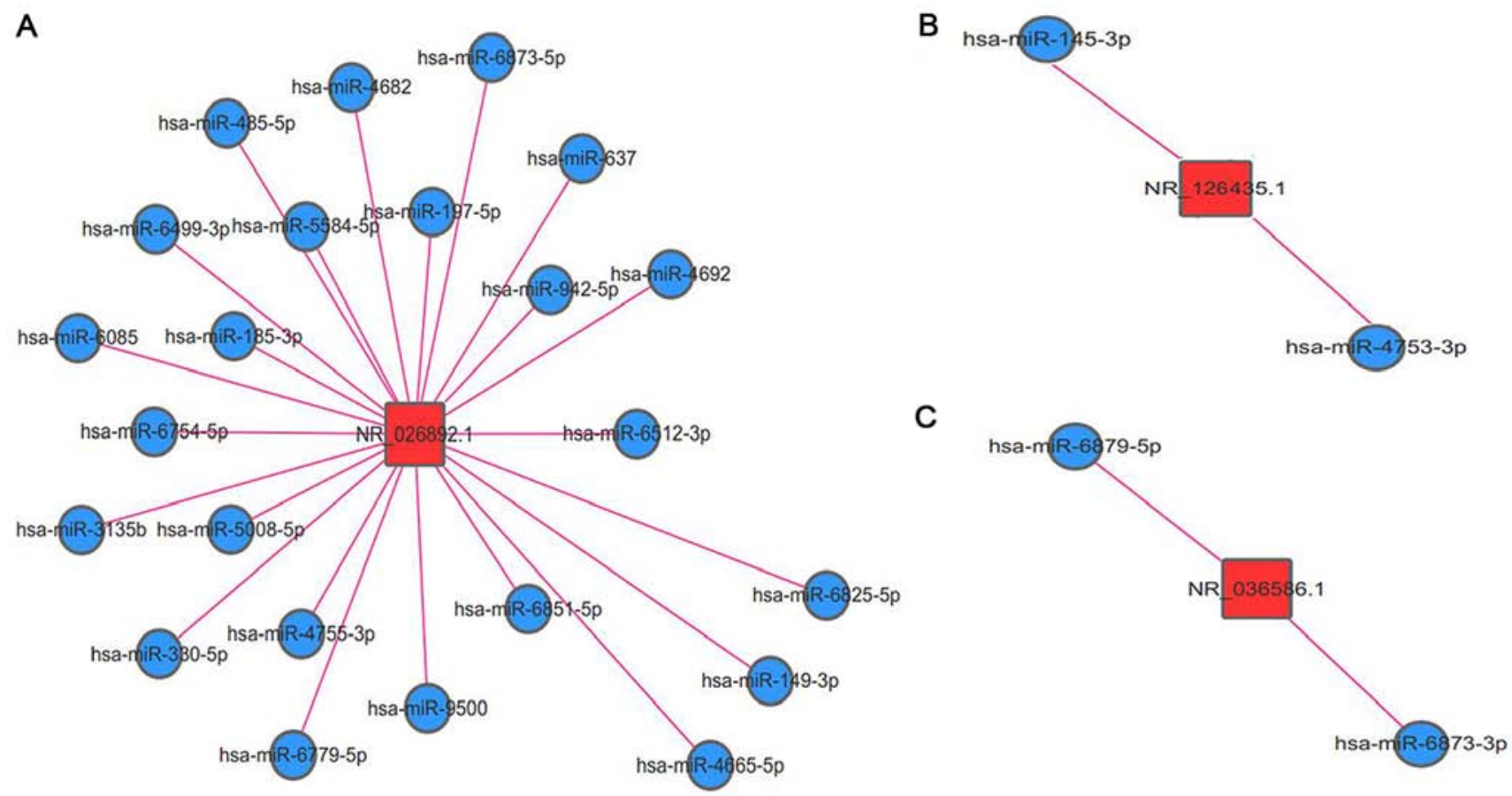

Figure 4. Network of three lncRNA-miRNA interactions. (A) NR_026892.1, (B) NR_126435.1 and (C) NR_036586.1. Red squares represent lncRNAs, blue circles correspond to mRNAs and pink lines refer to the target interaction of lncRNA-miRNA. IncRNA, long non-coding RNA; miRNA/miR, microRNA; hsa, Homo sapiens.
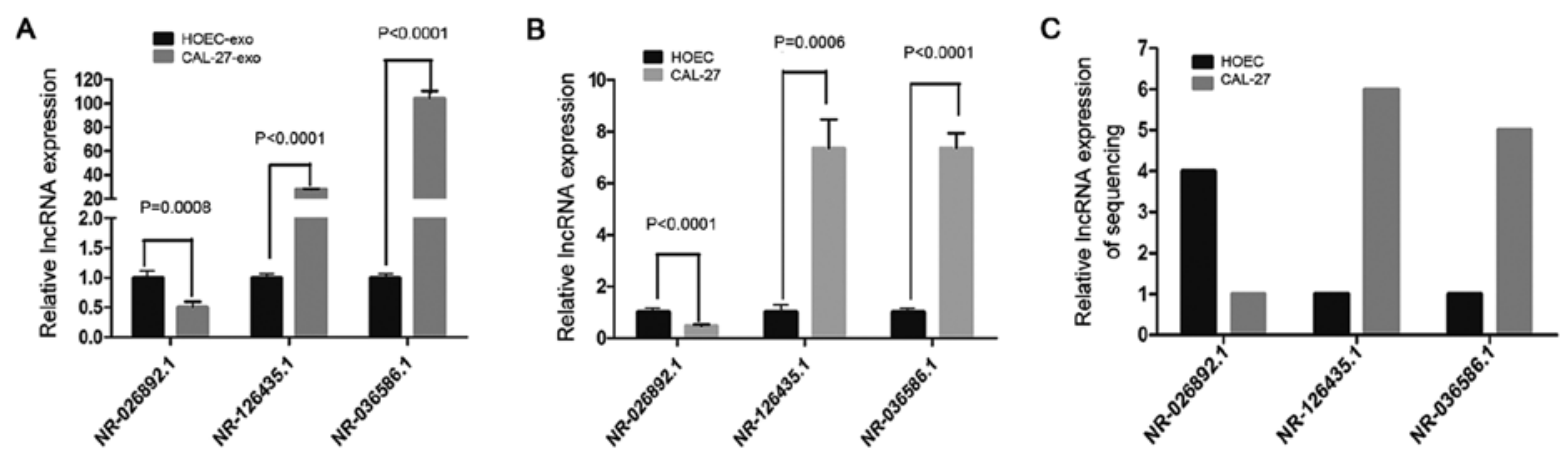

Figure 5. Relative expression levels of NR-026892.1, NR-126435.1 and NR-036586.1 in (A) exosomes (n=3), (B) cells ( $\mathrm{n}=3$ ) and (C) sequencing data (n=1). HOEC, human oral epithelial cell; exo, exosome; lncRNA, long non-coding RNA.

potential biomarkers of exo-lncRNAs. The lncRNA-miRNA interactions were also predicted to elucidate the functions of NR-026892.1, NR-126435.1 and NR-036586.1 (Fig. 4A-C, respectively). The network revealed that NR-026892.1 interacted with 23 miRNAs, whilst NR-126435.1 and NR-036586.1 interacted with 2 miRNAs each.

Relative expression levels of the three candidate IncRNAs in cells and exosomes. RT-qPCR analysis confirmed that the expression of the selected lncRNAs in CAL-27-exo was significantly different from that in HOEC-exo $(\mathrm{P}<0.001)$, and there were also significant differences between CAL-27 and HOECs $(\mathrm{P}<0.001)$. The relative expression levels of NR-026892.1 in CAL-27-exo and CAL-27 were approximately half of those in HOEC-exo and HOECs, respectively $(1.002 \pm 0.04328$ vs. $0.4784 \pm 0.02827$ in the cell group and $1.002 \pm 0.0451$ vs. $0.5022 \pm 0.05389$ in the exosome group), and the differences were significant (Fig. 5A and B). Conversely, the relative expression levels of NR-126435.1 and NR-036586.1 were markedly higher in CAL-27-exo and CAL-27 than in HOEC-exo and HOECs, respectively (Fig. 5A-C). For NR-126435.1, the overexpression was more marked in CAL-27-exo vs. HOEC-exo than in CAL-27 vs. HOEC $(1.001 \pm 0.02648$ vs. $27.77 \pm 0.3024$ in the exosome group and $1.009 \pm 0.09347$ vs. $7.358 \pm 0.6427$ in the cell group). NR-036586.1 was upregulated by nearly 100-fold in the exosomes of CAL-27 vs. HOEC and nearly 7-fold at the cellular level $(104.4 \pm 3.544$ vs. $1.001 \pm 0.03821 \mathrm{in}$ the exosome group and $7.104 \pm 0.4076$ vs. $1.001 \pm 0.033$ in the cell group). The RT-qPCR results were consistent with the sequencing data, which suggested that the results of the exosomal lncRNA sequencing were credible.

\section{Discussion}

Despite advances in treatment, the 5-year survival rate of patients with OSCC remains $<50 \%$ due to the lack of early diagnosis. To improve this situation, more sensitive and accu- 
rate biomarkers should be developed for the early detection of OSCC. According to The Cancer Genome Atlas, cancer initiation and progression are likely a result of the interaction of environmental factors and genetic alterations $(24,25)$. Evidence has demonstrated that lncRNAs modulate diverse processes in tumour progression, metastasis and suppression (26). Hence, a tumour may be detected in its early stage based on the evaluation of IncRNA expression levels in tissues. However, this procedure is limited due to the invasiveness of tissue collection. Of note, exosomal lncRNAs have a differential abundance in exosomes that may reflect the characteristics of parental cells (5), distinguishing certain exosomal lncRNAs as potential cancer markers.

Tumour exosomes (TEXs) have been extensively studied as novel biomarkers for the early detection and prognosis of cancer, as they may exist stably in the circulatory system. Signaling molecules, including RNAs and proteins, from exosomes may be targeted to cells; these cells may then produce various factors that are able to promote tumour proliferation, invasion and migration (27). TEX-IncRNA has an important utility in early cancer detection $(28,29)$.

Various OSCC cell lines, including OSC-4, SCC1, SCC2, SCC4, SCC9, CAL-27, UM1 and UM2, have been established. Continuously established OSCC cell lines have become important research tools to gain a better understanding of the pathogenesis of this disease and search for efficient therapeutic strategies. Amongst the cell lines mentioned above, CAL-27 is frequently used and regarded as a representative cell line in the field of OSCC. Jiang et al (30) selected CAL-27 to build OSCC models for in vitro and in vivo studies. While the in vitro studies have revealed satisfactory results, in vivo studies have demonstrated abnormal growth patterns of CAL-27 xenografts, with vesicles slowly growing at the surface and deeper areas of tumours. Jiang et al (30) concluded that certain established cell lines are not suitable for building OSCC models for in vitro and in vivo studies, as their growth patterns differ from one another. Therefore, CAL-27 cells were chosen to be used in the present study.

In the present study, KEGG analysis suggested that IncRNA-associated target genes were mostly enriched to the PI3K/Akt signalling pathway, which is a classic signalling pathway of cancer. Previous studies suggested that this pathway may be activated by numerous types of cellular stimuli or toxic insults and may regulate fundamental cellular functions, including transcription, translation, proliferation, growth and survival (31-35). Once activated, Akt may control key cellular processes by phosphorylating substrates involved in proliferation, metastasis and invasion. Therefore, differentially expressed lncRNAs may be involved in this cancer-associated pathway.

The function of lncRNAs is similar to that of miRNAs, which are able to regulate the expression of neighboring target genes $(36,37)$. The present study indicated that certain lncRNAs are significantly associated with their target genes and that those lncRNAs exert their function through their predicted target genes.

IncRNAs and target genes may interact in cis and trans forms. The target genes obtained by using the cis model must meet two criteria: i) Genes should be located in the range of $10 \mathrm{~kb}$ upstream and downstream of three significantly and differentially expressed exo-lncRNAs; ii) a significant co-expression trend should be observed in three exo-lncRNAs. In the trans model, target genes acquire the sequences of candidate exo-lncRNAs and differentially express mRNAs first. These sequences are then screened by consecutively using blast $\left(\mathrm{e}<1 \times 10^{-5}\right)$ and RNAplex $(\mathrm{G}<20)(38,39)$.

NR_126435.1, one of the selected lncRNAs, is located on chromosome 4 and its target genes are associated with fibroblast growth factor 5 (FGF5) and von Hippel-Lindau tumour suppressor (VHL). FGF5, identified as an oncogene, belongs to the FGF family. FGF family members are able to promote mitogenesis and cell survival, which are involved in various biological processes, including embryonic development, cell growth, angiogenesis, tissue repair, tumour growth and progression. FGF5 expression is markedly upregulated in patients with cancer $(40,41)$. VHL is able to form a multimeric complex that functions in the ubiquitination and degradation of hypoxia-inducible-factor (HIF). Exosomes derived from hypoxic OSCCs promote the migration and invasion of normoxic OSCCs via HIF- $1 \alpha$ and HIF- $2 \alpha(13,42)$. The interaction between NR_126435.1 and its target genes, e.g., FGF5 and VHL, implied that NR_126435.1 may participate in oncogenesis. NR_126435.1 was overexpressed in CAL-27 and CAL-27-exo (fold-change $>6, P=0.0006$ ). NR-026892.1 is located on chromosome 4 and its target genes are associated with multiple coagulation factor deficiency 2 (MCFD2), which is a marker of testicular germ cell tumours (43). Studies have demonstrated that MCFD2 promotes OSCC metastasis by regulating the expression levels of lectin, mannose binding 1 and galectin 3 binding protein. Hence, MCFD2 may be a promising novel therapeutic target in patients with metastatic OSCC (44). In the present study, the network of lncRNA-miRNA interactions revealed that NR-026892.1 interacted with 23 miRNAs. These miRNAs also have a role in numerous types of cancers. For instance, miR-485-5p is a potential tumour suppressor in different cancer types and its overexpression may inhibit SCC25 proliferation by PAK1 (45). miR-9500 is able to reduce the expression levels of Akt1 and affect tumourigenesis and metastasis (46). miR-637 acts as a tumour suppressor in pancreatic cancer cells (47). miR-149-3p targets Akt1 and induces cell apoptosis (48). In previous studies, Akt1, as a serine/threonine-protein kinase, was demonstrated to have anti-proliferative and anti-migratory effects and functioned as an oncogene in multiple types of cancer (46). These results suggested that NR-026892.1 may participate in cancer metastasis. NR_036586.1 is located in chromosome 2 and its target gene is associated with period circadian regulator 2 (PER2), whose gene polymorphisms may be closely linked to certain types of cancer. Downregulation of PER2, which is a promising predictor of OSCC, may contribute to the tumourigenesis and development of OSCC; PER2 may also regulate its anti-carcinogenic biological function by interacting with several signalling pathways, including the P53/cyclin dependent kinase inhibitor 2A, PIK3CA/AKT and caspase 8 pathways (49). Therefore, similar to NR_126435.1, NR_036586.1 might be prone to tumourigenesis.

In conclusion, to the best of our knowledge, the present study was the first to report that TEX-lncRNA may contribute to the early detection of OSCC based on bioinformatics analysis outcomes. NR-026892.1, NR-126435.1 and NR-036586.1 
may be explored as future detection tools through rigorous verification and mechanistic investigation. Further studies are warranted to conclusively determine whether the three candidate IncRNAs may be considered useful OSCC biomarkers. Therefore, in further studies by our group, exosomes will be isolated from pathological samples of OSCC and adjacent tissues and from salivary/blood specimens of patients with oral premalignant lesions and OSCC in the near future.

\section{Acknowledgements}

Not applicable.

\section{Funding}

The present study was supported, in part, by the National Natural Science Foundation of China (grant no. 31572522), Gansu Natural Science Foundation (grant no. 17JR5RA217) and the Fundamental Research Funds for the Central Universities (grant nos. lzujbky-2017-it44, lzujbky-2018-it44 and lzukqky-2019-t01).

\section{Availability of data and materials}

The datasets used and/or analyzed during the current study are available from the corresponding author on reasonable request.

\section{Authors' contributions}

$\mathrm{JJ}$ and $\mathrm{ZH}$ analyzed and interpreted the data regarding the exosomes and bioinformatics, and were major contributors in writing the manuscript. XLu, SW, MJ and XLi collected the clinical samples, performed the experiments and collected the experimental data. ZL and $\mathrm{XH}$ designed the experiments, reviewed and edited the manuscript. All authors read and approved the final manuscript.

\section{Ethics approval and consent to participate}

Human oral epithelial cells were isolated and used in accordance with the ethical standards established in the Declaration of Helsinki. The present study was approved by the Ethics Committee of the School/Hospital of Stomatology at Lanzhou University (Lanzhou, China) and informed consent forms were signed by the patients' parents prior to their participation in the study.

\section{Patient consent for publication}

Not applicable.

\section{Competing interests}

The authors declare that they have no competing interests.

\section{References}

1. Warnakulasuriya S: Global epidemiology of oral and oropharyngeal cancer. Oral Oncol 45, 309-316, 2009.

2. Shpitzer T, Hamzany Y, Bahar G, Feinmesser R, Savulescu D, Borovoi I, Gavish M and Nagler RM: Salivary analysis of oral cancer biomarkers. Br J Cancer 101: 1194-1198, 2009.
3. Sharma A, Kim JW and Paeng JY: Clinical analysis of neck node metastasis in oral cavity cancer. J Korean Assoc Oral Maxillofac Surg 44: 282-288, 2018.

4. McNamara KK, Martin BD, Evans EW and Kalmar JR: The role of direct visual fluorescent examination (VELscope) in routine screening for potentially malignant oral mucosal lesions. Oral Surg Oral Med Oral Pathol Oral Radiol 114: 636-643, 2012.

5. Colombo M, Raposo G and Théry C: Biogenesis, secretion, and intercellular interactions of exosomes and other extracellular vesicles. Annu Rev Cell Dev Biol 30: 255-289, 2014.

6. Yáñez-Mó M, Siljander PR, Andreu Z, Zavec AB, Borràs FE, Buzas EI, Buzas K, Casal E, Cappello F, Carvalho J, et al: Biological properties of extracellular vesicles and their physiological functions. J Extracell Vesicles 4: 27066, 2015.

7. Ge Q, Zhou Y, Lu J, Bai Y, Xie X and Lu Z: miRNA in plasma exosome is stable under different storage conditions. Molecules 19: 1568 1575, 2014.

8. Théry C: Exosomes: Secreted vesicles and intercellular communications. F1000 Biol Rep 3: 15, 2011.

9. Bonasio R and Shiekhattar R: Regulation of transcription by long noncoding RNAs, Annu Rev Genet 48: 433-455, 2014.

10. Rafiee A, Riazi-Rad F, Havaskary M and Nuri F: Long noncoding RNAs: Regulation, function and cancer. Biotechnol Genet Eng Rev 34: 153-180, 2018.

11. Fan Q, Yang L, Zhang X, Peng X, Wei S, Su D, Zhai Z, Hua X and $\mathrm{Li} \mathrm{H}$ : The emerging role of exosome derived non coding RNAs in cancer biology. Cancer Lett 414: 107-115, 2018.

12. Wang F, Li L, Piontek K, Sakaguchi M and Selaru FM: Exosome miR 335 as a novel therapeutic strategy in hepatocellular carcinoma. Hepatology 67: 940-954, 2018.

13. Li L, Li C, Wang S, Wang Z, Jiang J, Wang W, Li X, Chen J, Liu K, Li C, et al: Exosomes derived from hypoxic oral squamous cell carcinoma cells deliver miR 21 to normoxic cells to elicit a prometastatic phenotype. Cancer Res 76: 1770-1780, 2016.

14. Ye SB, Zhang H, Cai TT, Liu YN, Ni JJ, He J, Peng JY, Chen QY, Mo HY, Jun-Cui, et al: Exosomal miR $243 \mathrm{p}$ impedes $\mathrm{T}$ cell function by targeting FGF11 and serves as a potential prognostic biomarker for nasopharyngeal carcinoma. J. Pathol 240 329-340, 2016.

15. Liu T, Chen G, Sun D, Lei M, Li Y, Zhou C, Li X, Xue W, Wang $\mathrm{H}$, Liu $\mathrm{C}$ and $\mathrm{Xu}$ J: Exosomes containing miR 21 transfer the characteristic of cisplatin resistance by targeting PTEN and PDCD4 in oral squamous cell carcinoma. Acta Biochim Biophys Sin (Shanghai) 49: 808-816, 2017.

16. Gomes CC, de Sousa SF, Calin GA and Gomez RS: The emerging role of long noncoding RNAs in oral cancer. Oral Surg Oral Med Oral Pathol Oral Radiol 123: 235-241, 2017.

17. Jin N, Jin N, Bu W, Li X, Liu L, Wang Z, Tong J and Li D: Long non-coding RNA TIRY promotes tumor metastasis by enhancing epithelial-to-mesenchymal transition in oral cancer. Exp Biol Med (Maywood) 245: 585-596, 2020.

18. Oda D and Wastson E: Human Oral Epithelial Cell Culture I. Improved Conditions for Reproducible Culture in Serum Free Medium. In Vitro Cell Dev Biol 26: 589-595, 1990.

19. Sdek P, Zhang ZY, Cao J, Pan HY, Chen WT and Zheng JW: Alteration of cell cycle regulatory proteins in human oral epithelial cells immortalized by HPV16 E6 and E7. Int J Oral Maxillofac Surg 35: 653-657, 2006.

20. Moffatt-Jauregui CE, Robinson B, de Moya AV, Brockman RD, Roman AV, Cash MN, Culp DJ and Lamont RJ: Establishment and characterization of a telomerase immortalized human gingival epithelial cell line. J Periodontal Res 48: 713-721, 2013.

21. Brueffer C and Saal LH: TopHat-Recondition: A post-processor for TopHat unmapped reads. BMC Bioinformatics 17: 199, 2016.

22. Livak KJ and Schmittgen TD: Analysis of relative gene expression data using real-time quantitative PCR and the $2(-\Delta \Delta \mathrm{C}(\mathrm{T}))$ Method. Methods 25: 402-408, 2001.

23. Théry C: Exosomes secreted vesicles and intercellular communications. F1000 Biol Rep 3: 1-8, 2011.

24. Lee H, Palm J, Grimes SM and Ji HP: The Cancer Genome Atlas Clinical Explorer: A Web and Mobile Interface for Identifying Clinical-Genomic Driver Associations. Genome Med 7: 112, 2015.

25. Warnakulasuriya S: Global epidemiology of oral and oropharyngeal cancer. Oral Oncol 45, 309-316, 2008.

26. Guttman M and Rinn JL: Modular regulatory principles of large non coding RNAs. Nature 482: 339-346, 2012.

27. Milane L, Singh A, Mattheolabakis G, Suresh M and Amiji MM: Exosome mediated communication within the tumor microenvironment. J. Control Release 219: 278-294, 2015. 
28. Li Q, Shao Y, Zhang X, Zheng T, Miao M, Qin L, Wang B, Ye G, Xiao B and Guo J: Plasma long noncoding RNA protected by exosomes as a potential stable biomarker for gastric cancer. Tumour Biol 36: 2007-2012, 2015.

29. Zhao R, Zhang Y, Zhang X, Yang Y, Zheng X, Li X, Liu Y and Zhang Y: Exosomal long noncoding RNA HOTTIP as potential novel diagnostic and prognostic biomarker test for gastric cancer, Mol Cancer 17: 68, 2018.

30. Jiang L, Ji N, Zhou Y, Li J, Liu X, Wang Z, Chen Q and Zeng X CAL 27 is an oral adenosquamous carcinoma cell line. Ora Oncol 45: e204-e207, 2009.

31. Hu Q, Lin X, Ding L, Zeng Y, Pang D, Ouyang N, Xiang Y, and Yao H: ARHGAP42 promotes cell migration and invasion involving PI3K/Akt signaling pathway in nasopharyngeal carcinoma. Cancer Med 7 3862-3874, 2018

32. Zhong C, Chen Y, Tao B, Peng L, Peng T, Yang X, Xia X and Chen L: LIM and SH3 protein 1 regulates cell growth and chemosensitivity of human glioblastoma via the PI3K/AKT pathway. BMC Cancer 18: 722, 2018.

33. Martins F, de Sousa SC, Dos Santos E, Woo SB and Gallottini M: PI3K AKT mTOR pathway proteins are differently expressed in oral carcinogenesis, J. Oral Pathol Med 45: 746-752, 2016.

34. Hou T, Zhou L, Wang L, Kazobinka G, Chen Y, Zhang X and Chen Z: Leupaxin promotes bladder cancer proliferation, metastasis, and angiogenesis through the PI3K/AKT pathway. Cell Physiol Biochem. 47: 2250-2260, 2018.

35. Sukawa $Y$, Yamamoto $H$, Nosho K, Ito M, Igarashi $H$, Naito $T$, Mitsuhashi K, Matsunaga Y, Takahashi T, Mikami M, et al: HER2 expression and PI3K Akt pathway alterations in gastric cance. Digestion 89: 12-17, 2014.

36. Cabili MN, Trapnell C, Goff L, Koziol M, Tazon-Vega B, Regev A and Rinn JL: Integrative annotation of human large intergenic noncoding RNAs reveals global properties and specific subclasses. Genes Dev 25: 1915-1927, 2011.

37. Zhang G, Lan Y, Xie A, Shi J,Zhao H, Xu L, Zhu S, Luo T, Zhao T, Xiao Y and Li X: Comprehensive analysis of lncRNA chromatin interactions reveals lncRNA functions dependent on binding diverse regulatory elements. J Biochem 294: 15613-15622, 2019.

38. Kopp F and Mendell JT: Functional Classification and Experimental Dissection of Long Noncoding RNAs. Cell 172: 393-407, 2018

39. Petruk S, Sedkov Y, Riley KM, Hodgson J, Schweisguth F, Hirose S, Jaynes JB, Brock HW and Mazo A: Transcription of bxd noncoding RNAs promoted by trithorax represses Ubx in cis by transcriptional interference. Cell 127: 1209-1221, 2006.
40. Huang Y, Wang $\mathrm{H}$ and Yang Y: Expression of fibroblast growth factor 5 (FGF5) and its influence on survival of breast cancer patients. Med Sci Monit 24: 3524-3530, 2018.

41. Liu D, Zhang C, Li X, Zhang H, Pang Q, Wan A: MicroRNA-567 inhibits cell proliferation, migration and invasion by targeting FGF $_{5}$ in osteosarcoma. EXCLI J 17: 102-112, 2018.

42. Balamurugan K: HIF 1 at the crossroads of hypoxia, inflammation, and cancer. Int. J Cancer 138: 1058-1066, 2016.

43. Gashaw I, Dushaj O, Behr R, Biermann K, Brehm R, Rübben H, Grobholz R, Schmid KW, Bergmann M and Winterhager E: Novel germ cell markers characterize testicular seminoma and fetal testis. Mol Hum Reprod 13: 721-727, 2007.

44. Fukamachi M, Kasamatsu A, Endo-Sakamoto Y, Fushimi K, Kasama H, Iyoda M, Minakawa Y, Shiiba M, Tanzawa H and Uzawa K: Multiple coagulation factor deficiency protein 2 as a crucial component in metastasis of human oral cancer. Exp Cell Res 368: 119-125, 2018.

45. Lin XJ, He CL, Sun T, Duan XJ, Sun Y and Xiong SJ: hsa miR $4855 p$ reverses epithelial to mesenchymal transition and promotes cisplatin induced cell death by targeting PAK 1 in oral tongue squamous cell carcinoma. Int J Mol Med 40: 83-89, 2017.

46. Yoo JK, Jung HY, Lee JM, Yi H, Oh SH, Ko HY, Yoo H, Kim HR, Song H, Kim S and Kim JK: The novel miR 9500 regulates the proliferation and migration of human lung cancer cells by targeting Akt1. Cell Death Differ 21: 1150-1159, 2014

47. Xu RL, He W, Tang J, Guo W, Zhuang P, Wang CQ, Fu WM and Zhang JF: Primate specific miRNA 637 inhibited tumorigenesis in human pancreatic ductal adenocarcinoma cells by suppressing Akt1 expression, Exp Cell Res 363: 310-314, 2018

48. Si L, Xu L, Yin L, Qi Y, Han X, Xu Y, Zhao Y, Liu K and Peng J: Potent effects of dioscin against pancreatic cancer via miR 149 $3 \mathrm{P}$ mediated inhibition of the Akt1 signalling pathway. Br J Pharmacol 174: 553-568, 2017.

49. Xiong H, Yang Y, Yang K, Zhao D, Tang H and Ran X: Loss of the clock gene PER2 is associated with cancer development and altered expression of important tumor-related genes in oral cancer. Int J Oncol 52: 279-287, 2018.

This work is licensed under a Creative Commons Attribution-NonCommercial-NoDerivatives 4.0 International (CC BY-NC-ND 4.0) License. 